\title{
Ducts in the human nipple: structure, epithelial immunophenotypes, and relationships with clear cells of nipple epidermis (toker cells) M Currie and JJ Going*
}

Address: University of Glasgow, UK

Email: JJ Going* - going@udcf.gla.ac.uk

* Corresponding author

from 6th International Symposium on the Intraductal Approach to Breast Cancer Santa Monica, CA, USA. 19-21 February 2009

Published: 24 July 2009

BMC Proceedings 2009, 3(Suppl 5):SI2 doi:10.1186/1753-656I-3-S5-SI2

This abstract is available from: http://www.biomedcentral.com/I753-656I/3/S5/SI2

(c) 2009 Currie and Going; licensee BioMed Central Ltd.

It has been suggested that there are structurally distinct 'type A' and 'type B' ducts in human nipple. If so, properties such as their relative numbers, accessibility at the nipple surface, and relationships with the breast lobes they communicate with could have biological significance and practical relevance for the intraductal approach to breast cancer diagnosis and treatment. To investigate the twoduct-type hypothesis further we examined duct structure and duct epithelial immunophenotypes in the nipples of cancer mastectomies. Relationships between major nipple ducts and clear cells of nipple epidermis (Toker cells) were also explored.

Sections through the nipple duct bundle at the base of the papilla in 10 mastectomy nipples were immunostained for oestrogen and progesterone receptor; proliferation markers Ki-67 and mcm-3; Cox-2; E-cadherin; basal epithelial markers p63, CD10, keratins 5, 14, 17 and luminal epithelial markers keratins $7 / 8$ and 19. In 30 further cases sagittal (and some coronal) sections were immunostained for keratins 7/8, 14 and progesterone receptor. Duct perimeters were measured for all ducts in transverse sections of the nipple duct bundle in 15 cases.

We did not find unambiguous evidence supporting the two-duct-type hypothesis. Duct perimeters at the base of the papilla were variable but did not apparently define separate populations of larger and smaller ducts; nor could separate duct populations be recognised on their epithelial immunophenotype. However, while some ducts open directly into a funnel-shaped infundibulum, others apparently taper to a pinpoint lumen, and may cluster around a common opening.

Clear cells of nipple epidermis (Toker cells) were present in variable numbers in 31/40 cases, usually around duct openings on the nipple surface, sometimes show features suggesting possible locomotion, and are occasionally present in keratin plugs. Similarities between clear cells of nipple epidermis and mammary precursor cells have been noted previously; their location and possible motility suggest new avenues for research. 